\title{
USING AUDITORY DISPLAY TECHNIQUES TO ENHANCE DECISION MAKING AND PERCEIVE CHANGING ENVIRONMENTAL DATA WITHIN A 3D VIRTUAL GAME ENVIRONMENT
}

\author{
James Broderick, Dr Jim Duggan, Dr Sam Redfern \\ College of Engineering and Informatics, \\ National University of Ireland, Galway, \\ University Road, Galway City, Galway, Ireland \\ j.broderick4@nuigalway.ie
}

\begin{abstract}
When it comes to understanding our environment, we use all our senses. Within the study and implementation of virtual environments and systems, huge advancements in the quality of visuals and graphics have been made, but when it comes to the audio in our environment, many people have been content with very basic sound information. Video games have strived towards powerful sound design, both for player immersion and information perception. Research exists showing how we can use audio sources and waypoints to navigate environments, and how we can perceive information from audio in our surroundings. This research explores using sonification of changing environmental data and environmental objects to improve user's perception of virtual spaces and navigation within simulated environments, with case studies looking at training and for remote operation of unmanned vehicles. This would also expand into how general awareness and perception of dynamic 3D environments can be improved. Our research is done using the Unity3D game engine to create a virtual environment, within which users navigate around water currents represented both visually and through sonification of their information using Csound, a $\mathrm{C}$ based programming language for sound and music creation.
\end{abstract}

\section{RELATED WORK}

Before this project, work was done using the Unity 3D game engine to create a virtual environment for collaboration and visualization of marine environmental data [1]. The system created a replication of Galway Bay and visualized surface current data across the bay, displaying the direction and speed of currents across the bay each hour over a month-long period. The goal was to show how such a system would allow for the data to be easily perceived and understood by a variety of users, who would then be able to use the system for collaborative discussion and decision making. The environment is created from LIDAR data of the seabed, creating quite a realistic representation. The ocean current is then mapped onto its correct geographical location within the environment, allowing for more data and in greater varieties than simple surface currents to be added to the system over time. The Galway Bay Model and some theories and ideas have been carried over to this project.

There are some examples of sonification of environmental data, with focus on user perception of the data rather than navigation. In DoppelLab [2], a virtual recreation of MIT's Media Lab was created in Unity. The goal of this recreation was for simultaneous representation of

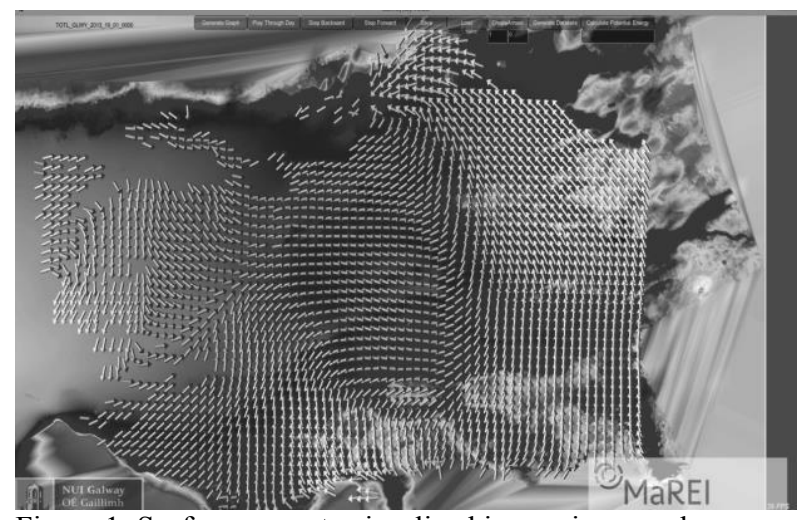

Figure 1: Surface currents visualized in previous work

environmental data captured by sensors within the building as visual and audio sources. With several hundred sensors around the building measuring humidity, temperature, noises levels, and actual captures of sound, it can be difficult to discern between different sources when relying purely on visual representations of data. The writer explored the usage of sonification to represent data as sound in addition to the visualizations. Being able to experience data both audibly and visually makes it easier for users to not only understand the environment, but easily discern where different sources of information end and begin, and how exactly they are located compared to each other. As users analyzed the virtual environment, they could better map out how busy different areas were at what times, how it affects temperature, etc. Seeing this, it is a natural to imagine that a same user could move from simply using this technique to understand the environmental data towards using this information to adjust their real-time actions or greater goal in the environment.

In fact, it has already been shown that, just like our reallife experiences, adding audio cues to our virtual environments aids our navigational ability. There have been several studies of using auditory navigation waypoints at specific goals or locations to aid users in being able to move through the environment [3][4]. Grohn and Lokki looked at measuring improvements in users finding objects within the environment using visual, audio, and audio visual cues to represent the target goals. While audio on its own was the least successful method of locating goal objects, combining visual and audio lead to users finding many more objects within a set time constraint. It was also found that users would use audio cues first to roughly locate an object before using visual stimuli for the final approach. Walker and Lindsay also looked at using auditory waypoints for navigation of an environment, specifically how users would 
be able to follow a path of waypoints with primarily auditory stimuli. It was found that users were well able to find and follow these waypoints, with the only common issue being users overshooting waypoints upon getting too close to them.

Both in terms of navigation and on a more complex scale, we are looking at aiding user decision making. Beyond examples of navigational decision making using auditory signals, there is also evidence of sonification aiding in other types of more complex decision making. Whether it be monitoring network traffic [5] or keeping track of multiple task prioritization [6][7], auditory display greatly enhances the user experience as a background process. Rather than distracting from the task at hand, users are simply listening for changes in their auditory background to indicate their attention is needed elsewhere. In situations where a purely visual display would hinder task completion and decision making, users can instead focus on the task at hand. Users were then not only able to handle their tasks at the best of their ability, but were better able to react to changing situations within multiple tasks simultaneously.

By better understanding user decision making, we can better create systems that aid users, especially when using new techniques for information perception. From studying instances of various navigational, task prioritization and task monitoring as seen earlier, it is understandable how exactly to fit in audio sources and measure their effectiveness in decision making. Beyond that, is important to understand how exactly users make decisions, especially as we hope to move on to more complex decision making experiments than simply navigational control. Some studies of decision making we looked at were the Beer Game, specifically with newer computerized versions of the game [8], and the Fish Banks Exercise [9]. While the Beer Game isn't as directly applicable to the modern world, it is still an interesting exercise and learning tool, especially for studying user's initial reactions and showing how small decisions cause greater effects on the rest of the system and users. It also allows students to see the importance of information gathering/sharing. The Fish Banks Exercise allows for similar studies of how poor short term decision making can damage the entire system. By using these studies and their research as to why users make the decisions they do, we hope to be able to carry over some of this knowledge to our own studies.

\section{HYPOTHESIS}

It's been shown by previously mentioned work that using static auditory beacons or waypoints, users are better able to navigate virtual environments. Users are also able to understand some amount of complex information represented as audio. The theory of this study is that by sonifying changing and mobile environmental data, we can aid in user navigation of these virtual environments. Rather than focusing on using auditory display for finding a user's destination, we want to look at how sonification of the potentially hazardous environment around the user can lead to better awareness of a user's location within an environment and their avoidance of hazards.

We use a reliance on hearing to keep us aware of how our environment outside of our sight changes, and this has been used in video games for a long time. Whether it be enemy footsteps, the sound of a power up activating, or even audio pings when an enemy draws close, games use auditory cues to keep users aware of their changing environment without cluttering the screen with a huge amount of visual information. Sound design such as this and the techniques that game developers use to portray this environmental information can be carried over to other virtual environments to better enhance user experience.

\subsection{Example Use Case}

A use case being examined is that of navigating a remotely operated vehicle (ROV) in a marine environment. Other researchers in the MaREI research group in the University of Limerick have worked on development of an ROV for marine missions, and as part were using a virtual control system for the ROV. As part of further research, they were moving this control system into a Unity 3D virtual environment and control system. When thinking of collaborative work between this and the previously mentioned Galway Bay virtual environment, we thought of how the control of the ROV could be enhanced with auditory display of parts of the environment. Visual aspects of the environment were already being displayed, but the presence of audio components could lead to users having a better perception of this remote, $3 \mathrm{D}$ environment.

As a first case study, this experiment will look at having users navigate a virtual rover through a marine environment. While the environment itself is a simple seabed based on LIDAR data of Galway Bay, water currents will provide the primary obstacle to navigation. These currents can change in intensity and direction, and can be in any location around the user. The aim is to sonify basic parameters of these currents so that the user has better awareness of their environment in all directions at all times, being able to tell the distance from, direction of, and strength of the currents around their position. This should lead to an improvement in navigation in timing or accuracy, as they will be less likely to stumble into a current outside of their field of view, or be surprised by a sudden change in direction or intensity. Since these currents can appear in any direction around them, sound should give better location awareness than just visual representation.

\section{PLANNED SYSTEM}

\subsection{Unity3D Game Engine}

Game engines have potential for use in non-entertainment projects for a variety of reasons. Modern game engines are built to be highly modular; where older engines were aimed at a specific type of game, modern engines are expected to be able to create anything from an independent 2D simulation game to a commercial first person shooter. They have basic components for cameras, 3D models, networking, controls, physics, etc., as well as having a huge amount of customization through creation of user scripts. All of this is aimed to make the engine applicable for a wider variety of games, but it also lets these tools be used for creation of nongaming projects, such as the collaborative visualization tool 


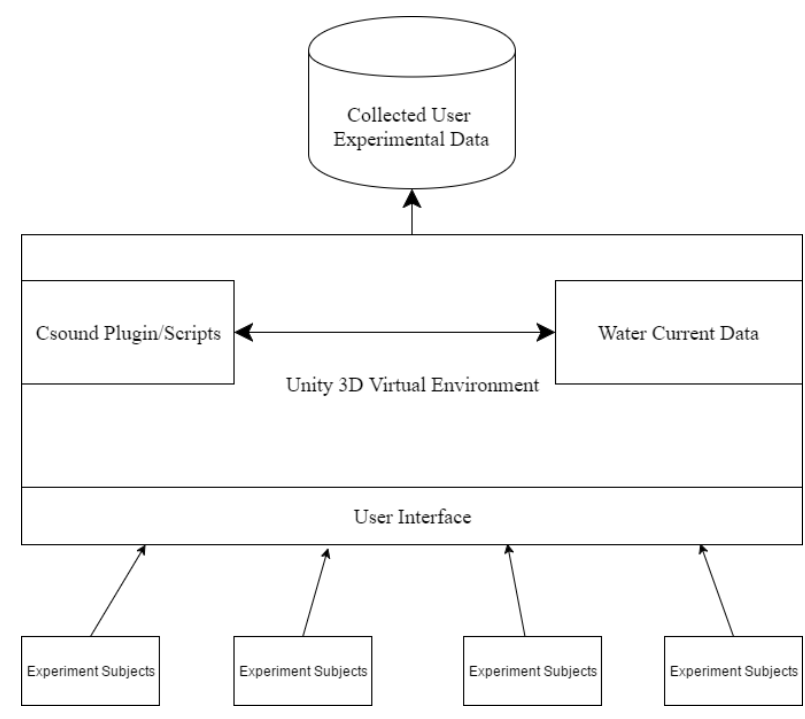

Figure 2: Architectural Diagram of planned system.

in this paper. By using a game engine for the basic setup of a system, more focus can be put on the actual functionality being created, rather than spending time recreating a rendering or physics engine. As well as this, with the growing popularity of independent games, many people are actively making tutorials and discussing game projects online, creating a wealth of information and experience.

The Unity3D game engine is the engine of choice for this research project, the reasons for which will be covered now. Most game engines provide a similar baseline functionality, with systems for rendering/visualization, sound, physics and scripting for functionality. Unity has a lot of flexibility with its scripting, as it allows for use of Unity versions of C\#, JavaScript and Boo. Scripts of different languages can be used in the same project and on the same game objects with little conflict. This means there is a smaller learning curve and gives more room for coders to work in their preferred language. Unity is also highly portable, easily able to deploy on platforms such as PC, OS $\mathrm{X}$, Linux, Mobile, Web, and a variety of game consoles. This portability means programs can be built to work on a variety of systems so that the maximum number of users can be supported. Unity has a huge user base, with people constantly writing tutorials, creating assets and answering questions. Additionally, projects and companies have used Unity for "Serious Games" in the past, including virtual environments [10], urban planning [11] and disaster simulation [12].

\subsection{Csound}

Csound is used for the auditory display requirements of the project. Csound is "a sound and music computing system" developed in 1985 at MIT Media Lab. It's a flexible way for creating computer driven music and sounds, running on a multitude of platforms. The primary reason for the usage of this sonification method is the existence of CsoundUnity, a C\# wrapper for Csound to be integrated with Unity3D. Developed by Rory Walsh [14], it allows for use of Csound based instruments and sounds within Unity environments, drawing information from the Unity environment for use by
Csound and extending Unity's audio API. Being able to have the modularity and flexibility of Csound directly available in Unity3D allows for a strong blend of visual and audio projects. While the sonification aspect of this first use case is relatively simple, having access to the strength of Csound means that similar projects can have a huge level of granularity when it comes to control of audio sources within their virtual environments. Hopefully by having free and easy access to a powerful 3D game engine that works directly with this software will allow for greater research into auditory display in virtual environments by new researchers.

\section{EXPERIMENTAL PROCESS}

The upcoming experimental process will involve users navigating an ROV through a series of currents in a Unity based virtual recreation of Galway Bay. Using mouse and keyboard controls like many generic video games, they will move the ROV through set checkpoints towards a destination. The possibility exists to also have the currents hinder or change the movement of the ROV, leading them to cause time issues as well as points penalties, but opening the possibility for smart use of the currents to save time. This gives user decision a greater depth of possibility, with more choices than simply what direction to go. While the users navigate the environment, the system can track user location data and movements, the time it takes them to reach objectives, and how often they stray into water currents, as well as the strength and direction of those currents. These factors can later be analyzed and compared.

Participants will be gathered primarily from within NUI Galway's Computer Science undergraduate classes. One known issue with auditory display is the additional learning element required. Users must be aware of what different audio means to gather useful information from it. By having mostly younger, computer-orientated participants, it is hoped that many of them will be used to basic gaming controls and functionality. This means that any learning within the environment can be focused on the auditory display side of the project. By reducing the amount of different simultaneous learning components, we can hopefully gather a truer reading of the usefulness of the sonification aspect.

As mentioned earlier, Unity's portability means the test environment can be deployed through the web. This planned experiment is aiming to use a web deployment of the system that users can connect to. Users access the test system through web browsers, $\log$ in with given details, and can perform the experiment entirely through their own machine with almost no setup. This means in addition to set computer

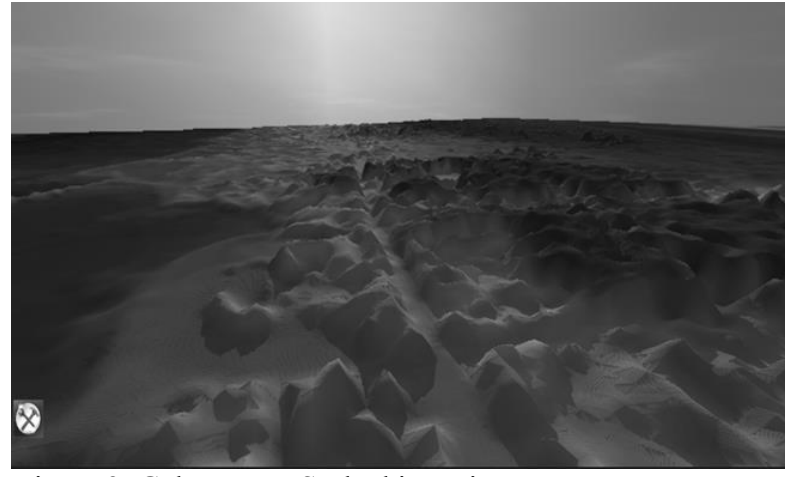

Figure 3: Galway Bay Seabed in Unity. 
lab settings, more data can be garnered through remote test groups who need only a reasonable computer setup, headphones, and an internet connection. User results are stored on the server for centralized access.

The ocean current data being used in the experiment is based off of surface currents of Galway Bay, expanded into a 3D grid. The original set of data is purely surface currents, containing location information, timestamps, and information on the water's direction, strength, etc. at that position. This data is converted into a grid across the bay by converting the real world latitude and longitude coordinates into coordinates within Unity space. This means that the currents are correctly positioned in their real world equivalent location within the virtual representation of Galway Bay. For the purposes of this experiment, this data is used as a basis for rough values due to it being 2 dimensional and having a distance of approximately $1 \mathrm{~km}$ between data points. The currents used in the virtual environment are exaggerated and more changeable than their real world counterpart to make the task more obvious for the user. However, with future work having access to more in-depth datasets and of a wider variety than ocean currents, the theory behind this experiment could be used for a wider set of case studies and ideas such as the ones listed previously.

The current information is sonified using three main parameters: Distance from the user, strength of the current, and direction of current. Using Unity's 3D sound placement, game objects in the virtual environment representing the currents will output audio sonifying the current it represents. The direction the sound appears to be coming from will adjust around the user, so users will hear currents from literally any direction around them. In addition to this, the volume of the sound grows as users get closer to the source of the audio. This means users will be able to tell what direction the current is, and roughly how close they are to it. After moving a certain distance from a sound source, audio is cut off to stop overwhelming of users with audio. The frequency of the audio changes depending on the speed of the current, so users will be able to tell how powerful a current is depending on how low or high the sound is. The combination of parameters should allow users to not only have a good idea of their full nearby surroundings, but also what directions and sounds should be prioritized. A more complicated parameter is sonifying the direction of the currents. As of now, this is using a moving sound source within the visual representation of the current. A simple Csound function is used to control the various parameters of the sound, with information on the current speed and distance from user handled by the Unity game object and fed into Csound as usable data.

One drawback of Unity's default audio is that its ability for audio spatialisation is limited. Currently it relies mostly on panning audio, which causes issues when audio sources are directly in front of or behind a user. These audio sources will sound identical, meaning users are forced to rotate their viewpoint to better orientate themselves with these sounds. This can be solved with implementation of Head Related Transfer Function (HRFT), which works to emulate how sounds are affected by our head and ears as we hear them. Part of our experimentation is to compare test groups using either form of audio spatialisation to see how great an effect it has on the user's perception of audio sources while multitasking within a virtual environment.

The system will have two main user types: Users who will move through the system with purely visual cues, and users who will receive a mixed audio-visual environment. There is no purely auditory level set as the goal of this research is to enhance visual systems with useful auditory displays. The first level of the system will have users trained in the system with only visual representation of currents in a simplified level. This allows users to focus on understanding the goals of the experiment and get used to the control of the ROV. The second stage will be a more complex current set up, and will also be the introduction of auditory representation of the nearby currents. This second stage is aimed at acclimatizing users to the sonification of the currents, and is primarily an additional training step. The third and final step is the primary source of results. Some users must navigate a level with only visual representations of the currents, and other users will have both visual representation and auditory representation. Performance in this step will be compared between user groups to find any improvement in navigation.

\section{PLANNED WORK}

The first stage of the experimental system is nearing completion. Once completed, experiments will be conducted with test groups to gather our first set of results. First will be examining changes to the current case study. Once user feedback and results have been gathered and analyzed, the method of sonifying can be further studied and improved. Different types of sound parameters may be used for sonifying environmental data in further experiments, and the specific values and distances can be fine-tuned. By using Csound and Unity, changing how the sound is represented, or how the environment handles the audio, are simple changes and can lead to more variety of experiments in the future.

There are also plans to implement support for Oculus Rift in the experiment to see if the addition of virtual reality and head tracking would have an effect on users' perception of the environment and ability to locate audio sources within the environment, as well as providing more in-depth information on how users move and react to changing sounds outside of their visual viewpoint. This would also make it easier for users to handle the back-front issue present when using simpler forms of audio spatialisation. As the experiment is built using Unity, the addition of Oculus Rift is relatively simple, with the main limiting factor being the additional equipment leading to smaller and slower testing of individuals.

There is also room for new types of experiments. Some plans are to look at combining previous work with auditory waypoints with the current system for environmental sonification. This can include measuring how additional sound objects affect user experience and understanding of the environment. As well as this, there is potential for having multiple types of environmental sonification that can be cycled through by the user in a single environment. Being able to switch between sonification of auditory hazards and audio waypoints showing directions to travel could have interesting results.

\section{ACKNOWLEDGMENT}

We would like to acknowledge the support of the Science Foundation of Ireland, the MaREI project and the College of Engineering and Informatics at NUI Galway. 


\section{REFERENCES}

[1] J. Broderick, J. Duggan, S. Redfern, "Using Game Engines for Marine Visualisation and Collaboration", in Proc of the 2016 Int. Conf. on Image, Vision and Computing (ICIVC), Portsmouth, UK, 2016, pp. 96-101.

[2] N. Joliat, "DoppelLab: Spatialized Data Sonification in a 3D Virtual Environment", Master's Thesis, Massachusetts Institute of Technology, February 2013, retrieved from http://hdl.handle.net/1721.1/85427

[3] B. Donmez., M. Cummings, \& H. Graham, "Auditory decision aiding in supervisory control of multiple unmanned aerial vehicles", Human Factors, Vol 51, Issue 5, pp 718-729, 2009

[4] D. Brock, J. Stroup, J. Ballas, "Effects of 3D Auditory Display on Dual Task Performance in a Simulated Multiscreen Watchstation Environment", Proceedings of the Human Factors and Ergonomics Society Annual Meeting, Vol. 46, Issue 17, pp 1570-1573, 2002.

[5] P. Kaminsky, D. Simchi-Levi, "A new computerized beer game: A tool for teaching the value of integrated supply chain management. Supply Chain and Technology Management”, pp, 216-225, 1998

[6] J. Whelan, "Building the fish banks model and renewable resource depletion", pp. 70, October 2001.

[7] S. Wang, Z. Mao, C. Zeng, H. Gong, S. Li, B. Chen, "A new method of virtual reality based on Unity3D", 18th International Conference on Geoinformatics, 2010, pp.1-5.

[8] A. Indraprastha, M. Shinozaki, "The investigation on using Unity3D game engine in urban design study", ITB Journal of ICT, vol. 3, issue 1, pp.1-18.

[9] S. Sharma, S. Jerripothula, S. Mackey, O. Soumare, "Immersive virtual reality environment of a subway evacuation on a cloud for disaster preparedness and response training", Proc. SPIE 9392, The Engineering Reality of Virtual Reality 2015, 939208 Attribution - Non Commercial 4.0 International License. The full terms of the License are available at http://creativecommons.org/licenses/by-nc/4.0/ 